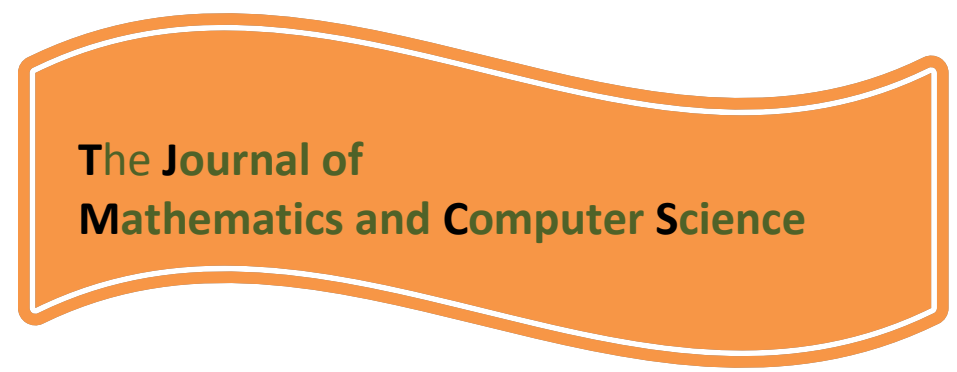

Available online at

\title{
http://www.TJMCS.com
}

The Journal of Mathematics and Computer Science Vol. 4 No.3 (2012) 456 - 462

\section{Hydrodynamic Modeling of Fluid body interaction by Fully Non-Linear Numerical Towing Tank}

\author{
Arash Abbasnia ${ }^{1, *}$, Mahmoud Ghiasi ${ }^{2}$, Seyed Mohammad Hossein Sharifi ${ }^{3}$ \\ Marine Technology Faculty of Amir Kabir University of Technology \\ a_abbasnia@aut.ac.ir \\ Marine Technology Faculty of Amir Kabir University of Technology \\ mghiasi@aut.ac.ir \\ Nautical Science Faculty of Petroleum University of Technology \\ Sharifi@put.ac.ir
}

Received: February 2012, Revised: May 2012

Online Publication: July 2012

\begin{abstract}
In basic design of offshore or onshore structures, prediction of surface waves due to uniform motion of the floating body is essential to achieve an optimum body shape. Whereas, in the practical hydrodynamics, using of towing tanks is common and so cost and time consuming to conduct it, so a reliable numerical tank is interesting. A wide channel with constant depth constitutescomputational domain. It assumes that fluid is incompressible and non-viscous and the flow is irrotational. Therefore, Laplace's equation could describe flow field.3D Boundary Element method based on second Green's Identityis implemented to solveLaplace's equation. Impermeable boundary condition is satisfied by Image method and Cauchy integral theorem and Poisson summation formula is used to determine Principle value integral. In this study, numerical simulation is conducted for a hemisphere and added mass and generated wave profile is presented.
\end{abstract}

Keywords: 3D Boundary Element Method, source, Green integral, Perturbation potential.

\footnotetext{
$1,{ }^{*}$ Corresponding author: Numerical Modeling of Marine Hydrodynamics

${ }^{2}$ Numerical Modeling and Computational Marine Hydrodynamics

${ }^{3}$ Head of Oil and Gas Research Center of Petroleum University of Technology
} 


\section{Introduction}

Boundary integral equation is defined in two schemes: direct and in-direct formulationsin order to analyze potential flow in several hydrodynamic problems. In-direct method is based on distributing sources on a body surface, whereas its strength is calculated by Fredholm's integral equation on body.Potential flow around submerged non-lifting bodies has been widely investigated by direct method. Itis based on the second Green's identity. Green function in this formulation is field's response due to a constant strength singularity presentswithin the uniform flow in the computational domain. Fundamental solution of a submerged source in a uniform flow is derived by Fourier transform approach. 3D Green function in finite depth was applied by Sahin and Hymann (1993, 1994). They used Cauchy Residue theorem and a adaptive quadratures set to evaluate principle value integral and determined fluid flow around axisymetric submerged body. To approximate resistance due to generated wave pattern of a wigley hull, Insel (1995) used a modified Green function in boundary integral equation and compared results with experimental measurements.

Accuracy of BEM depends on two items. First one is definition of body geometry and another one is order of distribution velocity potential over elements. Study on these items on the numerical solutions was conducted by Kouh and Ho (1996). In their research, Non-lifting body was discritized to flat and quadratic panels with constant and linear source strength distribution over theelements.

In addition, overlapping of source point and field point is a major difficulty in boundary integral implementation. Several remedies have been found for different problems. Cao (1991) was developed a numerical approach for direct and in-direct boundary integral based on Desingularzation distance for potential problems. Hwang (1998) used a subtracting and adding technique to desingularize boundary integrals for wave body interaction problem. Their approach is appropriate for open boundaries. To solve Laplace Equation in a closed domain, Grilli et al. (2001) summarize a formulation to remove singularity of direct boundary integral for curvilinear elements. Gao(2008) used curvilinear elements in desingularized in-direct method.

In this study, direct boundary integral equation is used to solve Laplace equation and isoparametric triangular element is chosen to prescribe boundary condition and geometry. Fully non-linear free-surface boundary condition is so complicated to be satisfied. By using Taylor expansion, free-surface boundary condition is satisfied in Green function and image method is used to apply impermeable bottom and walls tank.Total Velocity potential is divided in two parts. First part is uniform flow with constant velocity and second part is perturbation potential due to presentation of body in uniform flow. A new trick is presented to remove singularity of boundary integral implementation. Hydrodynamic performance of a hemisphere is considered to assess numerical procedure.

\section{Mathematical Formulation}

Consider a submerged or floating body in shallow water. The right hand coordinate system that is shown in Figure 1 is used. Its origin is located on undistributed free surface and $z$ axis is directed upward. It is assumed thatfluid flow is ideal, incompressible, steady and irrotational. The tank bottom is located at $z=-h$, and uniform flow with speed $U$ streams in negative $x$ direction. Consequently, the total steady-state velocity potential can be expressed as follow:

$$
\Phi(x, y, z)=-U x+\phi(x, y, z)
$$

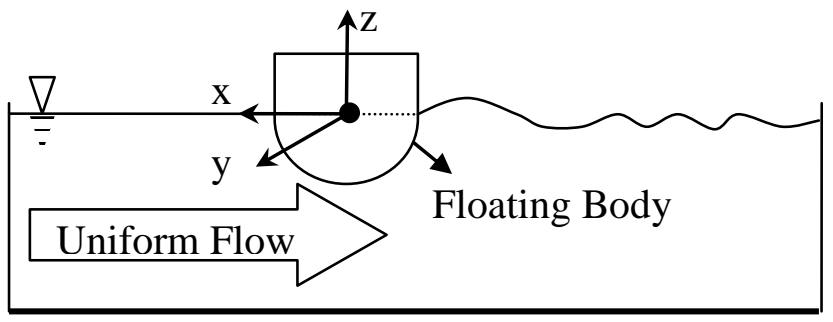

Figure 1.Definition Sketch 
Indeed, the total velocity potential includes uniform flow potential and perturbation potential. Whereas the uniform flow potential satisfies the Laplace's equation, the problem can be solved in term of perturbation potential. Thus, the governing equation can be written as:

$$
\nabla^{2} \phi=\frac{\partial^{2} \phi}{\partial x^{2}}+\frac{\partial^{2} \phi}{\partial y^{2}}+\frac{\partial^{2} \phi}{\partial z^{2}}=0
$$

To solve Equation 2, boundary conditions must be given which are categorized in the following:

- Bottom boundary condition

$$
\frac{\partial \phi}{\partial z}=0 \text { on } z=-h
$$

- Radiation condition

$$
\left\{\begin{array}{l}
\lim _{r^{2} \rightarrow \infty} \phi=O(1) \quad \text { for } \quad x<0 \\
\lim _{r^{2} \rightarrow \infty} \phi=0 \quad \text { for } \quad x>0
\end{array}\right.
$$

- Combined free surface boundary condition

$$
\frac{\partial^{2} \phi}{\partial x^{2}}+k_{0} \frac{\partial \phi}{\partial z}=0, \quad k_{0}=\frac{g}{U^{2}} \text { on } z=0
$$

- Hull Boundary condition

$$
\frac{\partial \Phi}{\partial n}=0 \quad \rightarrow \quad \frac{\partial \phi}{\partial n}=U n_{x} \text { on } S_{b}
$$

where, $S_{b}$ is body hull and $n_{x}$ is $x$ direction component of normal vector directed into fluid.

Second identity Green theorem is expressed as below:[3]

$$
\iint_{S_{q}}\left[\phi_{q} \frac{\partial G_{P q}}{\partial n_{q}}-G_{P q} \frac{\partial \phi}{\partial n_{q}}\right] d S=\varepsilon(P) \phi(P)
$$

where, $P$ is field point and $q$ is source point and $S_{q}$ indicates surface integral. $\varepsilon(P)$ depends on position of field point that can be located on smoothed body's surface $(\varepsilon(P)=2 \pi)$ or in control volume $(\varepsilon(P)=4 \pi)$ or out of domain $(\varepsilon(P)=0)$. The modified Green function for a source point at $\left(x_{0}, y_{0}, z_{0}\right)$ can be written as:

$$
G= \begin{cases}-\mu \sum_{n=-\infty}^{\infty}\left[\frac{1}{r_{1}^{\prime}}+\frac{1}{r_{1}^{\prime \prime}}+\frac{1}{r_{2}^{\prime}}+\frac{1}{r_{2}^{\prime \prime}}\right] & , \quad\left(x-x_{0}\right)>0 \\
-\mu \sum_{n=-\infty}^{\infty}\left[\frac{1}{r_{1}^{\prime}}+\frac{1}{r_{1}^{\prime \prime}}+\frac{1}{r_{2}^{\prime}}+\frac{1}{r_{2}^{\prime \prime}}\right]+\frac{16 \pi \mu}{W} \sum_{m=0}^{\infty} \frac{e^{-K_{m} h} \cosh \left(K_{m}\left(z_{0}+h\right)\right)\left(K_{0}+K_{m} \cos ^{2} \theta_{m}\right)}{K_{m} \cos \theta_{m}\left(1-K_{0} h \sec h^{2}\left(K_{m} h\right)+\sin ^{2} \theta_{m}\right)}, \quad\left(x-x_{0}\right)<0 \\
\frac{\cosh \left(K_{m}(z+h)\right)}{\cosh \left(K_{m} h\right)} \sin \left(K_{m}\left(x-x_{0}\right) \cos \theta_{m}\right) \times\left\{\begin{array}{l}
\cos \left(K_{m} y \sin \theta_{m}\right) \cos \left(K_{m} y_{0} \sin \theta_{m}\right) \\
\sin \left(K_{m} y \sin \theta_{m}\right) \sin \left(K_{m} y_{0} \sin \theta_{m}\right)
\end{array}\right.\end{cases}
$$

where, $\mu$ is strength of source point is taken unit and [5] 


$$
\begin{aligned}
& r_{1}^{\prime 2}=\left(x-x_{0}\right)^{2}+\left(y-y_{0}^{\prime}\right)^{2}+\left(z-z_{0}\right)^{2} \\
& r_{1}^{\prime \prime 2}=\left(x-x_{0}\right)^{2}+\left(y-y_{0}^{\prime \prime}\right)^{2}+\left(z-z_{0}\right)^{2} \\
& r_{2}^{\prime 2}=\left(x-x_{0}\right)^{2}+\left(y-y_{0}^{\prime}\right)^{2}+\left(z+\left(2 h+z_{0}\right)\right)^{2} \\
& r_{2}^{\prime \prime 2}=\left(x-x_{0}\right)^{2}+\left(y-y_{0}^{\prime \prime}\right)^{2}+\left(z+\left(2 h+z_{0}\right)\right)^{2}
\end{aligned}
$$

also

$$
\begin{aligned}
& y_{0}^{\prime}=y_{0}+2 n W \quad, \quad n=-\infty \ldots \infty \\
& y_{o}^{\prime \prime}=-y_{0}+(2 n+1) W \quad, \quad n=-\infty \ldots \infty
\end{aligned}
$$

where, $W$ is width of numerical tank. In the image method, the tank walls as mirror image singularity point in $y$ direction to infinity at both sides. Imaged sources' position can be described by $y_{0}^{\prime}$ and $y_{0}^{\prime \prime}$ in Equation 10.In Equation 8, sin term specified to odd $m$ and cos term specified to even $m$ which arises from Green function modification based on Cauchy integral theorem and Poisson summation formula. $\theta_{m}$ and $K_{m}$ in equation 10 are derived by solving two below equations at the same time.

$$
\begin{aligned}
& K_{m}-K_{0} \sec ^{2} \theta_{m} \tanh \left(K_{m} h\right)=0 \\
& K_{m} \sin \theta_{m}=m \pi / W
\end{aligned}
$$

\section{Desingularization Techniques}

Until now, various methods have been propounded to eliminate singular terms. In this paper, tricky approaches are proposed to obtain diagonal arrays of influence coefficient. In order to remove singularity, body's geometry surface is described by flat triangular panels $\left(S_{j}, j=1, \ldots N\right)$. Source points are located at center of each panel whereas; potential distribution over each element is constant. Figure 2 illustrates the location of field point and source points on the elements.

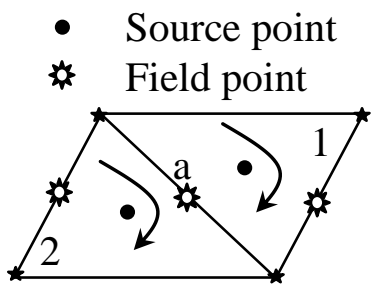

Figure 2.View of collocated source and field points

Field point (a) is positioned within the intersection edge of elements 1, 2. Field point potential can be defined by linear combination of velocity potentials of corresponding elements to each field points as below:

$$
\phi_{a}=\frac{\frac{\phi_{1}}{d_{1 a}}+\frac{\phi_{2}}{d_{2 a}}}{\frac{1}{d_{1 a}}+\frac{1}{d_{2 a}}}
$$

where,

$$
\begin{aligned}
& d_{1 a}=\sqrt{\left(x_{a}-x_{1}\right)^{2}+\left(y_{a}-y_{1}\right)^{2}+\left(z_{a}-z_{1}\right)^{2}} \\
& d_{2 a}=\sqrt{\left(x_{a}-x_{2}\right)^{2}+\left(y_{a}-y_{2}\right)^{2}+\left(z_{a}-z_{2}\right)^{2}}
\end{aligned}
$$


and $\phi_{1}, \phi_{2}$ is constant potential specified to the elements. It seems that potential value over the body surface is unknown boundary value and given boundary value is potential normal flux found as Neumann boundary condition. Therefore, descritized equation 7 can be written as:

$$
\sum_{j=1}^{N} \phi_{j} \iint_{S_{j}} \frac{\partial G_{i j}}{\partial n_{j}} d S_{j}-\varepsilon_{i} \frac{\frac{1}{d_{i m}}}{\frac{1}{d_{i m}}+\frac{1}{d_{i n}}} \phi_{m} \delta_{i m}-\varepsilon_{i} \frac{\frac{1}{d_{i n}}}{\frac{1}{d_{i m}}+\frac{1}{d_{i n}}} \phi_{n} \delta_{i n}=\sum_{j=1}^{N} U n_{x_{j}} \iint_{S_{j}} G_{i j} d S_{j}, i=1, \ldots, N
$$

where, $n$ and $m$ are corresponding panels to field points. $\delta$ isKronecker Delta Function. Gauss elimination method is used to solve linear algebraic system.

\section{Numerical Results}

A hemisphere is described by triangular flat elements is shown in Figure 3. In order to verify the present approach, surge added masses are achieved and compared with the analytical solutions for different number of mesh in table 1 . Radius of hemisphere is $r$ and uniform flow velocity is taken $1 \mathrm{~m} / \mathrm{s}$. Added mass is defined as:[6]

$$
m_{i j}=\rho \iint_{S_{b}} \phi_{j} \frac{\partial \phi_{i}}{\partial n} d S, \quad \mathrm{i}, \mathrm{j}=1,2, \ldots 6
$$

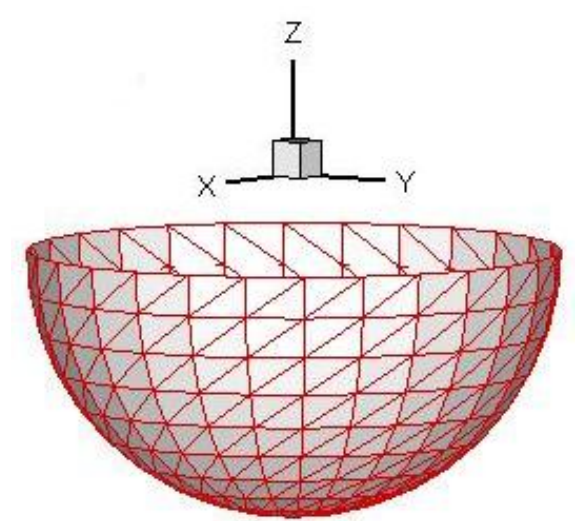

Figure 3.512 triangular elements over hemisphere

Table 1. Surge added mass of a sphere

\begin{tabular}{|l|c|c|}
\hline \multicolumn{1}{|c|}{ Case } & Number of Mesh & $m_{11} / \pi \rho r^{3}$ \\
\hline Case 1 & 256 & 0.356042 \\
\hline Case 2 & 528 & 0.346737 \\
\hline Case 3 & 756 & 0.335614 \\
\hline Case 4 & 1556 & 0.333453 \\
\hline Analytic & - & 0.333333 \\
\hline
\end{tabular}

Table 1 shows good agreements between numerical results and analytical results. In addition, for a tank

With $20 \mathrm{~m}$ depth and $30 \mathrm{~m}$ width, propagation wave due to uniform motion of hemisphere with different velocity is illustrated. 

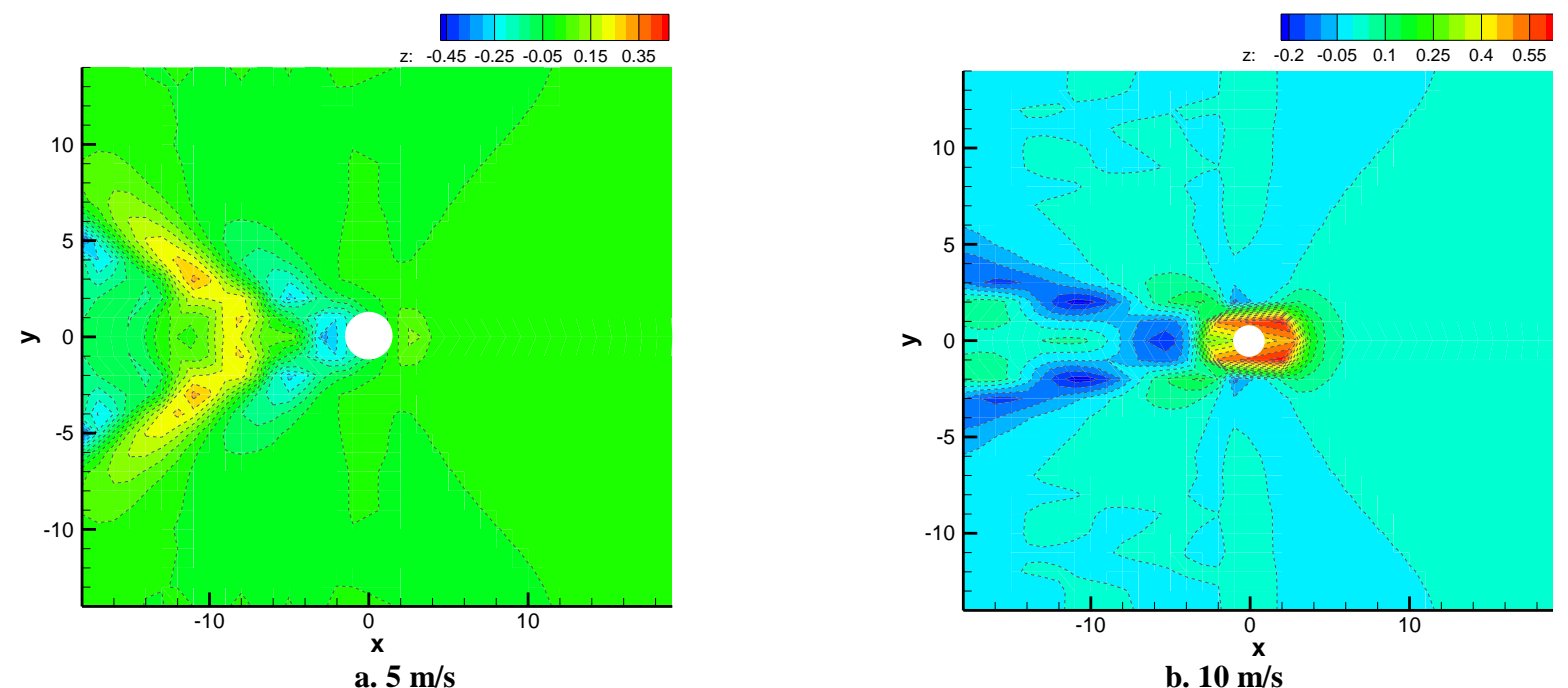

Figure 4.Wave Pattern due to uniform motion of a hemisphere

It seems that when velocity of body is increased, wave height is growing up and wave pattern is changed. This phenomenon is happened in the real world for floating marine structure. The higher wave height means higher resistance exerted by fluid to the body [2].In the far from the body, reflected wave from the walls tank is

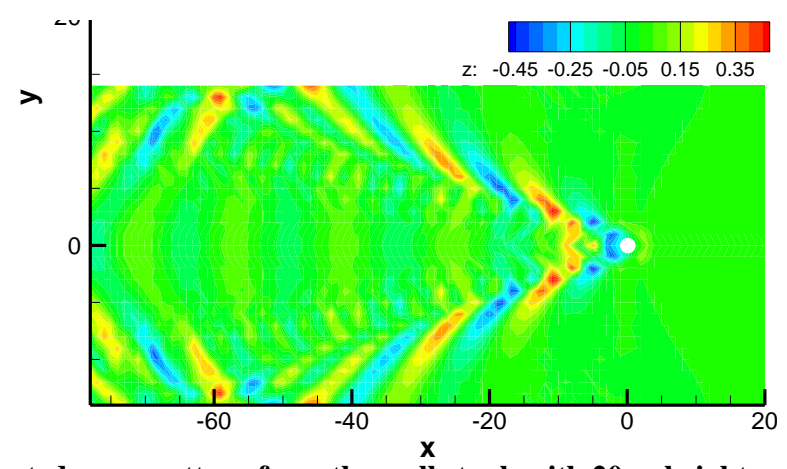

Figure 5.Reflected wave pattern from the walls tank with $20 \mathrm{~m}$ height and $30 \mathrm{~m}$ width

seen. Figure 5 shows reflected wave pattern due to the hemisphere with $5 \mathrm{~m} / \mathrm{s}$. it can be studied for different velocity.

\section{Conclusion}

In ocean engineering, determining resistance of floating marine structure is principal to gain the optimum hull design. Physical towing tank have been used to approximate wave making resistance for optimization process but it is so expensive and time consuming. In this paper, potential numerical towing tank based on potential theory and boundary elements methods is proposed instead of physical one. Direct boundary elements method is employed to solve boundary value problem. For BEM implementation, evaluating singular integral due to Green function is the mine difficulty. The new technique for flat triangle elements is presented for kernel surface integral. To verify the numerical results, surge added mass of a unit hemisphere is compared with analytical solution and in the following wave pattern for different velocity is illustrated to examine solver reliability. In general, presented procedure can be developed to use in applied marine hydrodynamics.

\section{References}

[1]Brebbia, C.A., "The boundary element method for Engineers", Wiley, 1978. 
[2]Faltinsen, O.M.,"Hydrodynamics of high-speed marine vehicles",Cambridge University Press, 2005. [3]Newman, J.N., "Marine Hydrodynamics",MIT Press,1989.

[4]Wehausen, J.V., and Laitone E.V., "Surface Waves",Online Copyright of University of California, 2002.

[5] Insel, M., and Doctors, L.J., "Wave-pattern prediction of monohulls and catamarans in a shallow water canal by linearized theory ", Proceeding of $12^{\text {th }}$ Australian Fluid Mechanics Conference, pp. 259-262, 1995.

[6] Gao, Z.L., and Zou, Z.J., "A three-dimensional desingularized high-order panel method based on NURBS", Journal ofHydrodynamics, Vol. 20, No. 2, pp. 137-146, 2008.

[7] Grilli, S.T., Guyenne, P., and Dias, F., "A fully non-linear model for three-dimensional overturning waves over an arbitrary bottom", International Journal forNumerical methods in fluids, Vol. 35, pp. 829-867, 2001.

[8] Hwang, W.S., "Boundary spectral methods for non-lifting potential flows", International Journal for Numerical methods in Engineering, Vol. 41, No. 6, pp. 1077-1085, 1998.

[9] Kouh, J.S., and Ho, C.H., "A high order panel method based on source distribution and gaussian quadrature", Journal ofShip technology, Vol. 43,pp. 38-47, 1996.

[10] Sahin, I., and Hyman, M., "Numerical calculation for the flow of submerged bodies under a free surface", Journal of Ocean Engineering, Vol. 20, No. 3, pp. 339-347, 1993.

[11] Sahin, I., Hyman, M., and Nguyen, T.C., "Three dimensional flow around a submerged body in finite-depth water", Journal of Applied Mathematical Modeling, Vol. 18, pp. 611-619, 1994.

[12] Cao, Y., "Computations of nonlinear gravity waves by a desingularized boundary integral method", Technical Report, The University of Michigan, 1991. 